\title{
Endotoxin removal in septic shock with the Alteco® LPS Adsorber was safe but showed no benefit compared to placebo in the double-blind randomized controlled trial - The ASSET study
}

Miklos Lipcsey ${ }^{1}$, Jyrki Tenhunen ${ }^{2}$, Soeren E. Pischke ${ }^{3,4}$, Anne Kuitunen $^{5}$, Hans Flaatten ${ }^{6}$, Lina De Geer ${ }^{7}$, Jan Sjölin ${ }^{8}$, Robert Frithiof ${ }^{2}$, Michelle S. Chew ${ }^{7}$, Stepani Bendel ${ }^{9}$, Rafael Kawati ${ }^{2}$, Anders Larsson ${ }^{10}$, Tom Eirik Mollnes ${ }^{11,12,13}$, Tor Inge Tønnessen ${ }^{14}$, Sten Rubertsson ${ }^{2}$.

${ }^{1}$ Hedenstierna laboratory, Department of Surgical Sciences/Anaesthesiology and Intensive Care Medicine, Uppsala University, Uppsala, Sweden.

${ }^{2}$ Department of Surgical Sciences/Anaesthesiology and Intensive Care Medicine, Uppsala University, Uppsala, Sweden.

${ }^{3}$ Division of Emergencies and Critical Care, Oslo University Hospital and

${ }^{4}$ Department of Immunology, University of Oslo, 0450 Oslo, Norway.

${ }^{5}$ Department of Intensive Care, University of Tampere and Tampere University Hospital, Tampere, Finland.

${ }^{6}$ Department of Clinical Medicine, Haukeland University Hospital, UiB Bergen, Norway.

${ }^{7}$ Department of Anaesthesiology and Intensive Care, and Department of Medical and Health Sciences, Linköping University, Linköping, Sweden.

${ }^{8}$ Section of Infectious Diseases, Department of Medical Sciences, Uppsala University, Uppsala, Sweden.

${ }^{9}$ Department of intensive care, Kuopio University Hospital, Kuopio, Finland.

${ }^{10}$ Section of Clinical Chemistry, Dept. of Medical Sciences, Uppsala University, Uppsala, Sweden. 
${ }^{11}$ Research Laboratory, Nordland Hospital, Bod $\varnothing$, and K.G. Jebsen TREC, University of Troms $\varnothing$, Norway.

${ }^{12}$ Department of Immunology, Oslo University hospital and University of Oslo, Norway.

${ }^{13}$ Centre of Molecular Inflammation Research, Norwegian University of Science and Technology, Trondheim, Norway.

${ }^{14}$ Division of Emergencies and Critical Care, Oslo University Hospital 0450 Oslo, and Institute of Clinical Medicine University of Oslo, 0316 Oslo, Norway. t.i.tonnessen@medisin.uio.no

${ }^{2}$ Sten Rubertsson, M.D., Ph.D., Department of Surgical Sciences/Anaesthesiology and Intensive Care Medicine, Uppsala University, Uppsala, Sweden. sten.rubertsson@surgsci.uu.se

\section{Corresponding author}

Miklos Lipcsey

Department of Surgical Sciences

Anaesthesiology and Intensive Care Medicine

Uppsala University

Akademiska sjukhuset

75185 Uppsala

Sweden

Phone: $+46-18-6171210$

Orcid-ID: 0000-0002-1976-4129 


\section{Competing interests}

The authors have disclosed that they do not have any conflicts of interest other than that stated below. MSC was Medical Advisor for Alteco AB and received reimbursement for her advice from 2014-2017. After this, she participated as clinical investigator. Moreover, Alteco AB financed the study and MSC, SR, ML, MSC, TIT, SP were reimbursed for travel expenses from the company.

\section{Funding}

The study is sponsored by institutional grants from Uppsala University Hospital, Uppsala University and by ALTECO Medical AB. RF was supported by funds from the Swedish Research Council (grant 523-2014-2569). Role of study Alteco Medical AB, Lund, Sweden: The study was designed; data collected, managed, analyzed, and interpreted by the investigators with the assistance of TFS Trial Form Support AB. The report was written by and the decision to submit the report for publication was taken by the investigators. The protocol and the final report were approved by the sponsor that did not have authority over the scientific content.

\section{Running head}

Endotoxin elimination in sepsis 


\section{Abstract}

\section{Purpose}

Lipopolysaccharides (LPS) are presumed to contribute to the inflammatory response in sepsis. We investigated if extracorporeal Alteco ${ }^{\circledR}$ LPS Adsorber for LPS removal in early Gram-negative septic shock was feasible and safe. Also, effect on endotoxin level, inflammatory response and organ function were assessed.

\section{Methods}

A pilot, double-blinded, randomized, Phase Ila, feasibility clinical investigation was undertaken in six Scandinavian intensive care units aiming to allocate thirty-two septic shock patients with abdominal or urogenital focus to LPS Adsorber therapy or a Sham Adsorber, therapy without active LPS-binding. The study treatment was initiated within 12 hours of inclusion and given for six hours daily on first two days. LPS was measured in all patients.

\section{Results}

The investigation was terminated after 527 days with eight patients included in the LPS Adsorber group and seven in the Sham group. Twenty-one adverse effects, judged not to be related to the device, were reported in three patients in the LPS Adsorber group and two in the Sham group. Two patients in the Sham group and no patients in the LPS Adsorber group died within 28 days. Plasma LPS levels were low without groups differences during or after adsorber therapy. The changes in inflammatory markers and organ function were similar in the groups.

\section{Conclusions}

In a small cohort of patients with presumed Gram-negative septic shock, levels of circulating endotoxin were low and no adverse effects within 28 days after LPS adsorber-treatment were observed. No benefit compared to a sham device was seen when using a LPS adsorber in addition to standard care. 
Trial registration: Clinicaltrials.gov NCT02335723. Registered: November 28, 2014

Keywords: Septic shock, Endotoxins, Hemoperfusion, Gram-Negative Bacteria 


\section{Introduction}

Septic shock is a life-threatening condition with profound systemic inflammatory activation triggered by infection (1). Despite management with antimicrobial therapy, source control and support of failing organs, mortality rates remain high (2). Therefore, the development of new treatment options for sepsis and septic shock is crucial for medical, humanitarian and health-economic reasons.

Gram-negative bacteria are a common cause of septic shock. Lipopolysaccharides (LPS), a group of endotoxins, are molecules found in the outer membrane of these bacteria. They are potent activators of the inflammatory system through the innate immune system and have been considered as one of the key triggers of the systemic inflammatory response (3-5). Hence, reducing the levels of LPS seems to be a logical and desirable strategy in the treatment of sepsis and septic shock. It is reasonable to hypothesize that using LPS adsorption membranes for the treatment of selected patients in the early phase of Gram-negative septic shock, within a strictly defined time-frame after the onset of clinical symptoms, may offer therapeutic benefits. Several extracorporeal endotoxin removal devices, based on various endotoxin-binding mechanisms, have been investigated with diverging results $(6-10)$.

Case reports from patients with septic shock suggest that endotoxin removal may be achieved in clinical practice with an LPS adsorber system (Alteco Medical AB, Lund, Sweden) with a high LPS affinity peptide binding mechanism, hereafter referred to as LPS Adsorber $(11,12)$. However, no randomized controlled trial has investigated the potential benefits of this LPS Adsorber.

We postulated that in patients with Gram-negative infections, early and extensive removal of LPS would limit the inflammatory response that characterizes septic shock. Unlike other device trials, the LPS Adsorber was compared to an identical Adsorber cartridge without active LPS-binding peptide. Thus, a double-blinded randomized sham -controlled pilot feasibility trial was performed. 
The primary endpoint was characterization of all reported adverse effects reported as Serious Adverse Device Effects (SADE) and Adverse Device Effects (ADE) as well as Serious Adverse Effects (SAE) and Adverse Effects (AE). Secondary endpoints were: changes in LPS levels during LPS Adsorber therapy; changes in the extent of organ failure; intensive care unit (ICU) mortality and 28-day mortality, ICU and hospital length-of-stay (LOS). We also measured plasma markers of the innate immune system, i.e. cytokines and complement activation as they are regarded as the first-line defense after bacterial infection. 


\section{Methods}

This was a pilot multicenter, stratified, parallel group, double-blinded, randomized, Phase lla, feasibility clinical investigation reported according the CONSORT and the SPIRIT guidelines $(13,14)$. The protocol has been reported previously (15).

The clinical investigation was conducted in compliance with applicable international standards (ISO 14155:2011), as well as with the ethical principles of the Declaration of Helsinki as adopted by the World Medical Association. The study was approved by local ethical review boards (Uppsala, Sweden; 2014/370, ALT C1-01; Norway, 2014/1059/REK vest; Tampere, Finland R14130).

The final protocol (6.0) was amended during the study on the $12^{\text {th }}$ of December, 2016.

Six general intensive care units (ICU) in Uppsala and Linköping, Sweden, Bergen and Oslo, Norway, and Tampere and Kuopio, Finland participated. We aimed at including 32 patients admitted to the ICU with confirmed septic shock after informed consent from the patient or legal representative. Recruitment of patients started in September 2015. Patients were stratified according to the origin of their suspected endotoxemia: 20 septic shock patients with abdominal focus (Stratum A) and 12 patients with urogenital focus (Stratum B). Patients were randomly allocated to standard care + LPS Adsorber therapy (LPS Adsorber group), or standard care + identical adsorber cartridge without active LPS-binding peptide (Sham group).

All inclusion and exclusion criteria are listed in the Supplemental Digital Content (SDC). A concise version is listed below. Upon enrolment (i.e. pre-treatment phase), patients admitted to the ICU with suspected endotoxemia were screened for fulfilment of the "Illness Severity Criteria" confirming early stage severe sepsis. Within six hours of enrolment, patients who fulfilled the "Treatment Criteria" confirming septic shock were randomized. 


\section{Illness Severity Criteria}

1) Patients must have suspected infection of abdominal or urogenital origin for which the patient is receiving intravenous antimicrobial therapy

2) Patients must have systemic inflammatory response syndrome (SIRS)(16)

3) At least one of the following criteria during the six hours prior to clinical investigation entry:

a) Metabolic acidosis

b) Acute oliguria/renal injury

c) Acute hepatic dysfunction

d) Thrombocytopenia

\section{Treatment Criteria}

4) SOFA score of 10 or higher (17), AND a Simplified Acute Physiology Score (SAPS II) of 58 or higher (18).

5) Patients must have received $\geq 30 \mathrm{~mL} / \mathrm{kg}$ of intravenous fluid within the six hours prior to randomization.

6) Vasopressor support for at least two hours prior to randomization to maintain mean arterial pressure $(\mathrm{MAP})>65 \mathrm{mmHg}$ or systolic arterial pressure $>90 \mathrm{mmHg}$.

7) The clinical investigation intervention initiated within 12 hours of fulfilment of the illness severity criteria.

\section{Randomization}

Patients were randomized with 1:1 ratio in blocks of four stratified by Stratum to either LPS Adsorber, or Sham groups by being treated with a randomly numbered blinded cartridge with the lowest serial number on site. Each site received therefore at least four blinded device packages per shipment. Study investigators, laboratory and research staff were blinded to treatment allocation and data until the analyses for the final report. Stratum A and Stratum B patients were kept apart by different serial numbers. 


\section{Intervention}

Figure S1 (SDC) summarizes the timeline of the clinical investigation. Treatment with LPS Adsorber or Sham adsorber initiated within six hours (Day 1) following fulfilment of the "Treatment Criteria" and given for six hours. Dalteparin or tinzaparin as bolus was used as anticoagulation during each treatment session. A second device treatment was performed 24 hours after the end of the first device treatment on Day 2. Treatment on Day 2 was not given if septic shock had resolved (e.g. no need for vasopressor support, new limitations of care). At least one treatment session with two hours of treatment was required to fulfill the treatment protocol. Patients were followed to 28 days after enrolment. Apart from the protocol for LPS or Sham adsorber therapy, management of the patients was at the discretion of the attend physician.

\section{Endotoxin analysis}

Endotoxin was analyzed with limulus amebocyte lysate assay (LAL; Pierce LAL Chromogenic Endotoxin Quantitation Kit, Thermo Scientific, Waltham, MA) measuring endotoxin activity as well as by high-performance liquid chromatography coupled with mass spectrometry (HPLC/MS) quantifying esterized 3-hydroxymyristate $(3 \mathrm{OH})$, the most abundant hydroxylated fatty acid of the lipid A moiety of endotoxin (19).

\section{Complement activation and Cytokines}

Complement activation was measured by the plasma terminal C5b-9 complement complex (TCC). Also, ten cytokines were measured: Interleukin (IL)-6, IL-8, IL-10, interferon gamma-induced protein 10 (IP-10), monocyte chemotactic protein 1 (MCP-1), macrophage inflammatory protein 1 beta (MIP1ß), tumor necrosis factor (TNF), IL-1 receptor antagonist (IL-1RA), Regulated on Activation, Normal T Cell Expressed and Secreted (RANTES) and eotaxin. See also the SDC.

\section{Organ failure}

Sequential Organ Failure Assessment (SOFA) score, renal function, liver function, circulatory support and respiratory support data were collected as reported in the results. 


\section{Data}

Data was collected by local research staff. Sites were monitored regularly by the clinical research organization (CRO; TFS AB, Lund, Sweden). Training, assessment of collected data, data storage and management were performed by the CRO.

\section{Statistics}

The statistical analysis plan was published prior to commencing the study (15). Since this was a pilot study and with no evaluation of primary performance variables, the sample size was chosen for practical reasons. The Full Analysis Set consists of all randomized patients who were randomized and was analyzed by intention to treat. Differences in parametric data were assessed by t-tests or mixed linear models, while nonparametric data were assessed by Mann-Whitney, Wilcoxon paired or Friedmans ANOVA tests as appropriate. Correlations were assessed with Spearman-Rank correlations. A $p<0.05$ was considered significant. Statistica ${ }^{\circledR} 13.2$ software (Statsoft, Tulsa, OK) and GraphPad Prism were used. Data are presented as median (interquartile range; IQR) unless stated otherwise. 


\section{Results}

The study was terminated prior to reaching the pre-specified number of patients due to a low inclusion rate after 527 days, when 15 patients had been enrolled, of whom eight randomized to the LPS Adsorber and seven to the Sham group. Eight patients completed the full treatment protocol with two treatments. The inclusion of patients is depicted in Figure 1.

Patient characteristics are presented in Table 1. Six of 15 patients had growth of Gram negative bacteria in blood. The microbiological cultures are presented in Table S1 (SDC). The most common verified source of infection was colon perforation followed by cholangitis in septic shock patients with abdominal focus.

\section{Safety and feasibility}

Including SAEs (SDC, Table S2), there were a total of 21 AEs in five patients (33.3\%): 3 patients in the LPS Adsorber group and 2 patients in the Sham group. No AEs were judged to be related to device treatment i.e. none were SADE or ADE.

Four patients in the LPS adsorber group and three patients in the Sham group had adsorber devices replaced due to clotting. One patient in each group discontinued the clinical investigation because of repeated clotting of the device. One patient in the LPS Adsorber group discontinued because a lack of need for further ICU care after the first treatment.

\section{Endotoxin levels in plasma}

Endotoxin (LPS) levels measured with the LAL assay were below the detection limit in almost all samples (data not shown). Quantification of endotoxin by measuring $3 \mathrm{OH}$ showed very low levels of endotoxin and no difference in endotoxin levels between patients treated with LPS and sham absorbers at 2 and 6 hours after the start of LPS adsorber therapy on day 1 and 2 (Figure 2). 


\section{Inflammatory response}

The complement activation marker, TCC, increased in a biphasic fashion during the time periods corresponding to the LPS and sham adsorber treatments without differences between the groups, consistent with an equal activation of complement by the two devices (Figure 2). The activation by the devices contributed more to the complement activation than the sepsis per se.

The 10 cytokines showed a marked inter-individual variation with no statistical significance between the groups (Figure 2). They showed distinctly different patterns during the observation period. TNF, IL-6, IL-1ra, IL-8 and MIP-1 $\beta$ were increased from start, and gradually declined to baseline levels. RANTES showed a similar pattern, except for a peak in the LPS adsorber group at 24 hours. IP-10 increased markedly from start to 24 hours and then declined. Eotaxin declined after start and then showed a patter similar to TCC with two peaks corresponding to the adsorption periods. MCP-1 and IL-10 stayed low during the whole observation period.

\section{Organ function}

The median pre-treatment SOFA score was 12 in both groups prior to randomization, that were similar between the groups during the study (Table 2). The extent of organ failure expressed as Urine output per day, Cystatin C estimated glomerular filtration rate (eGFR cyst $_{\text {) }}$ ) Plasma bilirubin, Vasopressor dependency index, Arterial Lactate levels, $\mathrm{PaO}_{2} / \mathrm{FiO}_{2}$ ratio, and Blood platelet count during the first two days of the study were also similar between the groups. Although most of these variables improved during the first two days, platelets decreased in both groups during this period $(p<0.05)$. Renal replacement therapy free days were $23(12-28)$ vs. 12 (1-26), vasopressor free days 24 (20-26) vs. 24 (21-26), and ventilator free days (up to 28 days) were 21 (20-27) vs. 23 (19-25), for the LPS Adsorber group vs. Sham group, respectively ( $p=n$.s. for all). $30 \mathrm{H}$ did not correlate to SAPS, SOFA score, $\mathrm{PaO} 2 / \mathrm{FiO} 2$ ratio, Vasopressor dependency index, Arterial Lactate, Blood platelet count, Plasma bilirubin, or Cystatin C eGFR cyst $_{\text {at }}$ at the start of treatment 1. 


\section{Outcome}

Two patients, one male and one female, died within 28 days in the Sham group (after eight and 10 days), whereas no patients died in the LPS Adsober group. ICU LOS was 11 (6-14) days in the LPS Adsorber group and 11 (7-27) in the Sham group. One from the LPS Adsorber group (24 days) and one from the Sham group (22 days) were discharged from hospital before 28-days.

\section{Discussion}

The aim of this double-blinded randomized pilot study was to investigate the safety, feasibility, and potential biological and clinical effects of the specific, high affinity, high capacity LPS Adsorber system $(11,12)$. The investigation was terminated prematurely due to low inclusion rate, with less than half of the pre-set number of patients included. LPS Adsorber treatment was safe in this small cohort of patients, however technical problems, specifically clotting, was very common leading to abrogated adsorber treatments. Plasma endotoxin levels were low both before and after treatment, and were not different in the LPS adsorber and the Sham groups. The extent of inflammatory reaction, organ dysfunction and outcome were similar in the LPS adsorber and the Sham groups.

Given that this is a pilot study and the pre-set number of patients was not reached, the conclusions from the study are limited. Yet, several findings are of interest for further studies using the LPS Adsorber and for studies in other anti-LPS strategies. Importantly, the study shows that doubleblinded device studies are feasible in the critical care setting.

Patients undergoing LPS adsorber therapy did not experience more adverse effects compared to the Sham group. However, our data suggests that the adsorption procedure induced complement activation as measured by TCC, platelet consumption and eotaxin increase in both groups.

Although LPS levels in plasma have been reported to be increased during the course of sepsis (6), both in vitro and clinical data suggests that endotoxin levels are highest in early phase of septic shock $(20,21)$. This study was thus designed assuming that LPS adsorption would be most beneficial 
immediately after the initial dose of antibiotics was administered. Yet, we found low LPS levels despite inclusion criteria aiming at identifying patients with high endotoxin levels. Endotoxin levels were below the detection limit with LAL and low with HPLC/MS method (22). A reason for this could be that endotoxin levels decreased after the initial dose of antibiotics prior to LPS adsorber treatment similarly to a previous report (21). Alternatively, given the low mortality, despite the preset SAPS II and SOFA requirements, the endotoxin levels may have been low due to low degree of acute illness. A breakdown of LPS in the samples cannot be excluded. However, blood samples were handled according to our standard laboratory procedures, and we have previously reported detectable endotoxin levels from experimental research with these methods $(23,24)$.

The levels of endotoxin estimated by $3 \mathrm{OH}$ were low and did not decrease during LPS Adsorber therapy. This raises the question if extracorporeal LPS-adsorber therapies add substantially to endogenous endotoxin elimination. Although there are reports that imply decreased endotoxin levels with the endotoxin adsorber used in our study $(11,12)$, experimental and in vitro data have challenged all currently used LPS-adsorber therapies $(23,25)$.

Previous large randomized controlled studies on extracorporeal endotoxin elimination (6-10) included patient cohorts with variable illness severity. We aimed to include patients with high severity of illness with a predicted 28 day mortality over $40 \%$ setting high SAPS II and SOFA requirements for inclusion $(26,27)$. However, despite reaching the preset illness severity scores the mortality in our study was $13 \%$ at 28 days. Both patients who died received sham adsorber treatment while no patients died in the LPS Adsorber group. The occurrence of death to the Sham group does not yield a statistical difference and given the low number of patients in the study, even if there was difference the fragility index of this investigation would be very low (28).

Another aim of the study was to start LPS removal as early as possible after identifying an eligible patient. We experienced substantial difficulties in finding patients with a relatively specific group of pathologies. Specifically, capturing patients not responding to initial resuscitation (thus presumed to 
have high mortality), obtaining informed consent in these patients, then initializing a complex therapy within a short timeframe were logistical challenges. This problem is underlined by the decrease of most inflammatory mediators in our study irrespective of LPS Adsorber or sham treatment was given, suggesting that any intervention aimed at removing endotoxin or inflammatory mediators would need to be instituted very early during the course of septic shock, preferably with first dose of antibiotics. Alternatively, endotoxin removal therapies should be directed at a group with already high pre-treatment endotoxin levels, that we were unable to identify using clinical criteria alone.

\section{Strengths and Limitations}

Unlike previous studies (6-10), as far as we know, this is the first double-blinded extracorporeal endotoxin elimination study, which was made possible with a sham cartridge without LPS adsorber properties. Other obvious strengths of the study are the extensive clinical and laboratory data collected, including the analysis of inflammatory markers as well as the follow-up to 28 days.

On the other hand, early termination significantly limits the power of this study. However, one aim of this pilot study was to test the protocol for a possible phase IIb trial. With the current inclusion rate in six large centers several additional years would have been required to finalize the study. Another limitation of the study was that only one patient with urogenital sepsis was included. Urogenital sepsis is caused in the majority of cases by Gram-negative bacteria (29). Although septic shock is not uncommon in this group of patients, illness severity scores in this group were below inclusion levels. A further consequence of the early termination is that the characteristics of the patients at inclusion differed in some aspects, limiting the validity of findings that are based on group differences.

The study was designed to identify patients with Gram-negative sepsis, but not all patients presented with these bacteria according to culture results. Although the aim of the study was to decrease high levels of endotoxin in the blood related to Gram-negative infection, endotoxemia is present in sepsis 
of other etiologies too (30), suggesting that LPS adsorption therapy could have been of benefit even in patients with other types of severe infections.

\section{Future studies}

Based on this and other studies the role of endotoxin removal in sepsis is uncertain $(10,13)$. Any future study must identify patients with high endotoxin levels. Moreover the endotoxin removal should be started early with high capacity and specific endotoxin adsorbers.

\section{Conclusions}

In a small cohort of patients with presumed Gram-negative sepsis, no adverse effects of LPS adsorber-treatment were observed within 28 days after treatment, but it did not offer any clinical benefit compared to a sham device. The low level of circulating endotoxin suggests that antiendotoxin strategies are unlikely to give the desired benefit in this selected group of patients. 


\section{List of abbreviations}

\begin{tabular}{|c|c|}
\hline $\mathrm{AE}$ & Adverse effects \\
\hline ALT & Alanine aminotransferase \\
\hline AST & Aspartate aminotransferase \\
\hline CIP & Clinical investigation plan \\
\hline CRO & Clinical research organization \\
\hline CRRT & Continuous renal replacement therapy \\
\hline CT & Computed tomography \\
\hline eCRF & Electronic case report form \\
\hline eGFR cyst $_{\text {t }}$ & Estimated glomerular filtration rate based on Plasma Cystatin C \\
\hline HIT II & Heparin-induced thrombocytopenia II \\
\hline HPLC-MS & High-performance liquid chromatography coupled with mass spectrometry \\
\hline ICU & Intensive care unit \\
\hline IMD & Interventional medical device \\
\hline LAL & Limulus amebocyte lysate assay \\
\hline LOS & Length-of-stay \\
\hline MAP & Mean arterial pressure \\
\hline $\mathrm{PaO}_{2} / \mathrm{FiO}_{2}$ & Arterial oxygen tension/Fraction of inspired oxygen \\
\hline RRT & Renal replacement therapy \\
\hline SAE & Serious adverse effects \\
\hline SAPS II & Simplified acute physiology score \\
\hline SIRS & Systemic inflammatory response syndrome \\
\hline SOFA & Sequential organ failure assessment score \\
\hline
\end{tabular}




\section{Declarations}

\section{Ethical Approval and Consent to participate}

The clinical investigation was conducted in compliance with applicable international standards (ISO 14155:2011) and regulatory requirements, as well as with the ethical principles of the latest revision of the Declaration of Helsinki as adopted by the World Medical Association. The study has been approved by Regionala etikprövningsnämnden i Uppsala, Sweden (Regional Ethical Review Board in Uppsala; 2014/370, ALT C1-01), Regionale Komiteer For Medisinsk og Helsefaglig Forskningsetikk, Norway (Regional Committees for Medical and Health Research Ethics; 2014/1059/REK vest) and Tampereen Yliopistollisen Sairaalan Erityisvastuualueen Alueellinen Eettinen Toimikunta, Finland (Regional Ethical Committee of the Tampere University Hospital; R14130). Each patient was consented according to the regulations of the country where the patient is included in the study. The signed informed consent form was obtained by the Investigator prior to inclusion in the study. Should subjects be incapable of giving informed consent (e.g., subjects may be unconscious or obviously disoriented), the Clinical Investigator requested informed consent from the legally acceptable representative(s). As soon as the subject's medical condition allowed, he/she was informed about the clinical trial, and asked to provide informed consent for continued participation. Participation in the clinical investigation is voluntary and subjects (or their legally accepted representatives) could discontinue their participation at any time.

\section{Authors' contributions}

ML drafted the manuscript based on the protocol. ML and JT contributed equally to the protocol. AL, SP and TEM planned and conducted the laboratory investigations. All authors contributed to and approved the manuscript. ML, JT, RF, JS and SR designed the study and wrote the protocol with contributions from MSC, RK, TIT, HF, AK and SB. SR is the principal investigator. Site investigators are TIT, HF, AK, LDG and SB. 
Acknowledgements

We thank the TFS Trial Form Support AB for editing the protocol, running the study and for keeping/managing data. 


\section{References}

1. Ziesmann MT, Marshall JC: Multiple Organ Dysfunction: The Defining Syndrome of Sepsis. Surg Infect (Larchmt) 19 (2): 184-190, 2018.

2. Rhodes A, Evans LE, Alhazzani W, Levy MM, Antonelli M, Ferrer R, Kumar A, Sevransky JE, Sprung $\mathrm{CL}$, Nunnally ME, et al: Surviving Sepsis Campaign: International Guidelines for Management of Sepsis and Septic Shock: 2016. Intensive Care Med 43 (3): 304-377, 2017.

3. Annane D, Bellissant E, Cavaillon JM: Septic shock. Lancet 365 (9453): 63-78, 2005.

4. Lipcsey M, Larsson A, Eriksson MB, Sjölin J: Inflammatory, coagulatory and circulatory responses to logarithmic increases in the endotoxin dose in the anaesthetised pig. J Endotox Res 12 (2): 99-112, 2006.

5. Suffredini AF, Fromm RE, Parker MM, Brenner M, Kovacs JA, Wesley RA, Parrillo JE: The cardiovascular response of normal humans to the administration of endotoxin. N Engl J Med 321 (5): 280-287, 1989.

6. Bengsch S, Boos KS, Nagel D, Seidel D, Inthorn D: Extracorporeal plasma treatment for the removal of endotoxin in patients with sepsis: clinical results of a pilot study. Shock 23 (6): 494-500, 2005.

7. Payen DM, Guilhot J, Launey Y, Lukaszewicz AC, Kaaki M, Veber B, Pottecher J, Joannes-Boyau O, Martin-Lefevre L, Jabaudon M, et al: Early use of polymyxin B hemoperfusion in patients with septic shock due to peritonitis: a multicenter randomized control trial. Intensive Care Med 41 (6): 975-984, 2015.

8. Cruz DN, Antonelli M, Fumagalli R, Foltran F, Brienza N, Donati A, Malcangi V, Petrini F, Volta G, Bobbio Pallavicini FM, et al: Early use of polymyxin B hemoperfusion in abdominal septic shock: the EUPHAS randomized controlled trial. JAMA 301 (23): 2445-2452, 2009.

9. Klein DJ, Foster D, Walker PM, Bagshaw SM, Mekonnen H, Antonelli M: Polymyxin B hemoperfusion in endotoxemic septic shock patients without extreme endotoxemia: a post hoc analysis of the EUPHRATES trial. Intensive Care Med 44 (12): 2205-2212, 2018.

10. Dellinger RP, Bagshaw SM, Antonelli M, Foster DM, Klein DJ, Marshall JC, Palevsky PM, Weisberg LS, Schorr CA, Trzeciak S, et al: Effect of Targeted Polymyxin B Hemoperfusion on 28-Day Mortality in Patients With Septic Shock and Elevated Endotoxin Level: The EUPHRATES Randomized Clinical Trial. JAMA 320 (14): 1455-1463, 2018.

11. Ala-Kokko TI, Laurila J, Koskenkari J: A new endotoxin adsorber in septic shock: observational case series. Blood Purif 32 (4): 303-309, 2011.

12. Duszynska W, Smiechowicz J, Adamik B, Zielinski S, Kubler A: Advanced therapeutic methods for the treatment of meningococcal septic shock - case report. Anaesthesiol Intensive Ther 44 (4): 212216, 2012.

13. Chan AW, Tetzlaff JM, Altman DG, Dickersin K, Moher D: SPIRIT 2013: new guidance for content of clinical trial protocols. Lancet 381 (9861): 91-92, 2013.

14. Schulz KF, Altman DG, Moher D, Group C: CONSORT 2010 statement: updated guidelines for reporting parallel group randomised trials. BMJ 340 c332, 2010.

15. Lipcsey M, Tenhunen J, Sjolin J, Frithiof R, Bendel S, Flaatten H, Kawati R, Kuitunen A, Tonnessen TI, Rubertsson S: Abdominal Septic Shock - Endotoxin Adsorption Treatment (ASSET) - endotoxin 
removal in abdominal and urogenital septic shock with the Alteco(R) LPS Adsorber: study protocol for a double-blinded, randomized placebo-controlled trial. Trials 17 (1): 587, 2016.

16. Bone RC, Balk RA, Cerra FB, Dellinger RP, Fein AM, Knaus WA, Schein RM, Sibbald WJ: Definitions for sepsis and organ failure and guidelines for the use of innovative therapies in sepsis. The ACCP/SCCM Consensus Conference Committee. American College of Chest Physicians/Society of Critical Care Medicine. Chest 101 (6): 1644-1655, 1992.

17. Vincent JL, Moreno R, Takala J, Willatts S, De Mendonca A, Bruining H, Reinhart CK, Suter PM, Thijs LG: The SOFA (Sepsis-related Organ Failure Assessment) score to describe organ dysfunction/failure. On behalf of the Working Group on Sepsis-Related Problems of the European Society of Intensive Care Medicine. Intensive Care Med 22 (7): 707-710, 1996.

18. Le Gall JR, Lemeshow S, Saulnier F: A new Simplified Acute Physiology Score (SAPS II) based on a European/North American multicenter study. JAMA 270 (24): 2957-2963, 1993.

19. Pais de Barros JP, Gautier T, Sali W, Adrie C, Choubley H, Charron E, Lalande C, Le Guern N, Deckert V, Monchi M, et al: Quantitative lipopolysaccharide analysis using HPLC/MS/MS and its combination with the limulus amebocyte lysate assay. J Lipid Res 56 (7): 1363-1369, 2015.

20. Crosby HA, Bion JF, Penn CW, Elliott TS: Antibiotic-induced release of endotoxin from bacteria in vitro. J Med Microbiol 40 (1): 23-30, 1994.

21. Brandtzaeg P, Kierulf P, Gaustad P, Skulberg A, Bruun JN, Halvorsen S, Sorensen E: Plasma endotoxin as a predictor of multiple organ failure and death in systemic meningococcal disease. $J$ Infect Dis 159 (2): 195-204, 1989.

22. Weil D, Pais de Barros JP, Mourey G, Laheurte C, Cypriani B, Badet N, Delabrousse E, Grandclement E, Di Martino V, Saas P, et al: Circulating levels of 3-hydroxymyristate, a direct quantification of endotoxaemia in noninfected cirrhotic patients. Liver Int 39 (1): 106-114, 2019.

23. Carlsson M, Lipcsey M, Larsson A, Tano E, Rubertsson S, Eriksson M, Sjolin J: Inflammatory and circulatory effects of the reduction of endotoxin concentration in established porcine endotoxemic shock--a model of endotoxin elimination. Crit Care Med 37 (3): 1031-e1034, 2009.

24. Thorsted A, Bouchene S, Tano E, Castegren M, Lipcsey M, Sjolin J, Karlsson MO, Friberg LE, Nielsen El: A non-linear mixed effect model for innate immune response: In vivo kinetics of endotoxin and its induction of the cytokines tumor necrosis factor alpha and interleukin-6. PLoS One 14 (2): e0211981, 2019.

25. Harm S, Falkenhagen D, Hartmann J: Endotoxin adsorbents in extracorporeal blood purification: do they fulfill expectations? Int J Artif Organs 37 (3): 222-232, 2014.

26. Beck DH, Smith GB, Pappachan JV, Millar B: External validation of the SAPS II, APACHE II and APACHE III prognostic models in South England: a multicentre study. Intensive Care Med 29 (2): 249256, 2003.

27. Jones AE, Trzeciak S, Kline JA: The Sequential Organ Failure Assessment score for predicting outcome in patients with severe sepsis and evidence of hypoperfusion the time of emergency department presentation. Crit Care Med 37 (5): 1649-1654, 2009.

28. Ridgeon EE, Young PJ, Bellomo R, Mucchetti M, Lembo R, Landoni G: The Fragility Index in Multicenter Randomized Controlled Critical Care Trials. Crit Care Med 44 (7): 1278-1284, 2016. 
29. Chung VY, Tai CK, Fan CW, Tang CN: Severe acute pyelonephritis: a review of clinical outcome and risk factors for mortality. Hong Kong Med J 20 (4): 285-289, 2014.

30. Wang T, Cui YL, Lin ZF, Chen DC: Comparative Study of Plasma Endotoxin with Procalcitonin Levels in Diagnosis of Bacteremia in Intensive Care Unit Patients. Chin Med J (Engl) 129 (4): 417-423, 2016. 


\section{Supplemental Digital Content (SDC)}

Supplemental Digital Content file ASSET.docx

\section{Figure legends}

Figure 1. CONSORT diagram

Figure 2. LPS levels, complement activation (TCC) and 10 cytokines during LPS Adsorber and sham treatment of septic patients. Red lines represent LPS adsorber group, green lines represent Sham adsorber group. All values are displayed as median (line in the box), mean (dot) and interquartile range (box) and $10-90^{\text {th }}$ percentile (whiskers). No group differences were seen. Abbreviation of cytokines are as described in Materials and Methods.

\section{Tables}

Table 1 and 2 are submitted in separate files. 